\title{
First Principle Study of Structural and Electronic Properties of $\mathrm{CBe}_{2}$ Compound
}

\author{
K. GrusZKA* \\ Częstochowa University of Technology, 42-201 Częstochowa, Poland \\ Doi: 10.12693/APhysPolA.139.617 \\ ${ }^{*}$ e-mail: konrad.gruszka@pcz.pl
}

\begin{abstract}
In this paper, a DFT-based study of the structural and electronic properties at ambient pressure of pure beryllium carbide $\left(\mathrm{CBe}_{2}\right)$ phase is presented. The PBE-GGA approach, as implemented in the Quantum Espresso electronic structure code, was used through the scalar-relativistic calculation. A structural equilibrium parameters and bulk modulus were also calculated. Obtained results show that phase is semiconducting in nature with a band gap equal to $1.22 \mathrm{eV}$. Electronic properties in terms of band structure and density of states are presented. Calculations show that magnetic ordering is not favorable and total magnetic moment is zero. Using electron localization function and the Bader charge analysis we also determined a bonding character inside $\mathrm{Be}_{2} \mathrm{C}$.
\end{abstract}

topics: electronic structure, DFT, semiconductor

\section{Introduction}

Beryllium carbide in its pure form is a colorless transparent crystal historically identified by Lebeau [1] with $\mathrm{Be}_{2} \mathrm{C}$ composition. For a long time, only one crystalline form of this compound was known, the so-called anti-fluorite structure, previously studied and experimentally reported by Wycoff [2]. Recently, some additional possible structures of beryllium carbide have been identified to be energetically stable, including quasi planar hexacoordinate carbon [3], a promising 2D material, or a stable hexa-coordinate monolayer [4]. A wide range of applications for beryllium carbide such as for ablation coatings in experimental fusion reactors [5] or as the diffusion barrier against atomic copper [6] has recently drew attention to this material. However, among many experimental and theoretical articles $[7-10]$ related to $\mathrm{Be}_{2} \mathrm{C}$, according to the author's best knowledge, there is no theoretical work devoted to the study of electronic properties of this compound.

The chemical composition of beryllium carbide in its bulk form may suggest that the compound should be characterized by low mass and at least insulating or semiconducting behavior, due to its transparency in the visible part of the electromagnetic spectrum. The available literature seems to confirm these assumptions, at least for lowdimensional (2D) structures where ab-initio data were previously published [3, 4]. In [3], $\mathrm{Li}$ et al. identified two types of electronically stabilized $\mathrm{Be}_{2} \mathrm{C}$, the quasi-planar hexa-coordinate $\left(\mathrm{Be}_{2} \mathrm{C}-\mathrm{I}\right)$ and tetra-coordinate $\left(\mathrm{Be}_{2} \mathrm{C}-\mathrm{II}\right)$ indicating that the former form is more stable in terms of energy. Then, in the work of Maseri et al. [4], a new polycrystalline two-dimensional form of this material was reported, namely $\mathrm{Be}_{2} \mathrm{C}$-III. However, although interesting, known two-dimensional systems may have different properties than $\mathrm{Be}_{2} \mathrm{C}$ in its bulk form. The formation mechanism of beryllium carbide was also studied using molecular dynamics (MD) simulations of the sputtering process $[11,12]$ and tested for absorption and diffusion in the context of operating the material under reactor conditions [13]. Therefore, in order to fill the gap of bulk $\mathrm{CBe}_{2}$ material, in this paper we present DFT-based first principles theoretical calculations focusing on structural and electronic properties of this interesting compound.

\section{Computational details}

The calculations were performed using a Quantum Espresso software package [14]. Structural relaxation and electronic structure calculations were performed using the plane wave method using the generalized gradient approximation (GGA) in the Perdew, Burke, and Ernzerhof (PBE) form. For all calculations, the projector augmented-wave (PAW) pseudopotentials were used. The electronic configurations for $\mathrm{Be}$ and $\mathrm{C}$ were $1 s^{2} 2 s^{2}$ and $1 s^{2} 2 s^{2} 2 p^{2}$, respectively. The $\mathrm{CBe}_{2}$ crystallizes in the face centered cubic structure with the $F m-3 m$ space group, where one can distinguish two sublattices: the carbon fcc and internal SC Be sublattice. Together they form the anti-fluorite structure, which is presented in Fig. 1. 


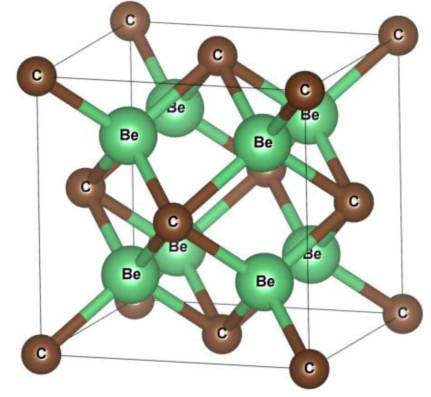

Fig. 1. Cell structure of $\mathrm{CBe}_{2}$.

All computations were done including the scalar relativistic effects. For ensuring accurate results, proper convergence tests were conducted. The Monkhorst-Pack uniform $k$-point mesh $15 \times 15 \times 15$ for the Brillouin zone sampling was established on that base. The energy cutoff for the wavefunction kinetic energy is $E_{\text {cut,kin }}=62 \mathrm{Ry}$ and the cutoff for the charge density is $E_{\text {cut }, \rho}=248 \mathrm{Ry}$. The electronic convergence criterion was set to $10^{-8} \mathrm{Ry}$. For the relaxation procedures, a $10^{-4} \mathrm{Ry} /$ atom convergence criterion was used for ionic minimization. This criterion was met when all components of all forces were less than this value. The above parameters used during calculations were carefully checked by utilising proper convergence tests.

\section{Results}

In the first step, the relaxation of both atomic positions as well as cell parameters optimization were done using the classic minimization of energy in respect to the fcc lattice $a$ parameter. The resulting structure is presented in Fig. 1. From energy in respect to the volume curve obtained in this step, the equilibrium lattice parameter equal to $a=4.33 \AA$ was obtained and the bulk modulus equal to $K=180.4 \mathrm{GPa}$ was calculated using the Murnaghan equation of state [15], i.e.,

$$
E(V)=\frac{E_{0} K_{0} V}{K_{C}^{\prime}}\left(\frac{\left(V_{0} / V\right)^{K_{0}^{\prime}}}{K_{C}^{\prime}-1}+1\right)-\frac{K_{0} V_{0}}{K_{C}^{\prime}-1},
$$

where $K_{0}$ - the bulk modulus, $K_{0}^{\prime}$ - the derivative of $K_{0}, E_{0}$ - the equilibrium energy, $V_{0}$ - the equilibrium volume and $V$ - the volume.

After ionic relaxation of the structure, a selfconsistent field (SCF) calculation of the total energy was conducted including breaking of magnetic symmetry to form the ferromagnetic case. During the SCF cycles, the introduced magnetic moment was always quenched to zero, regardless of the site on which it was induced, indicating that the material is not susceptible to magnetization. Next, the band structure along with the density of states was calculated and presented in Fig. 2.

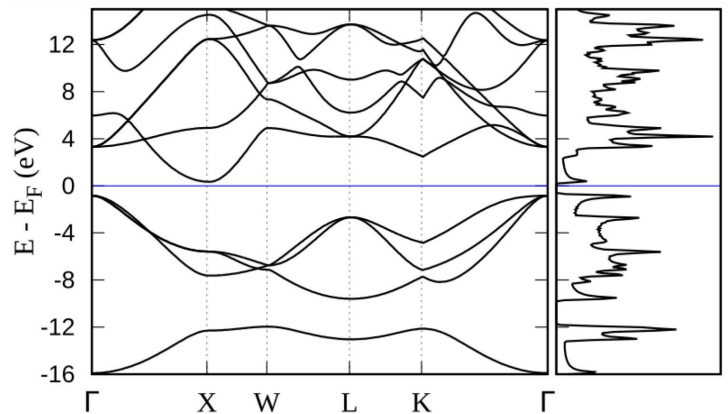

Fig. 2. Band structure and density of states of $\mathrm{CBe}_{2}$. The blue line indicates the Fermi level.

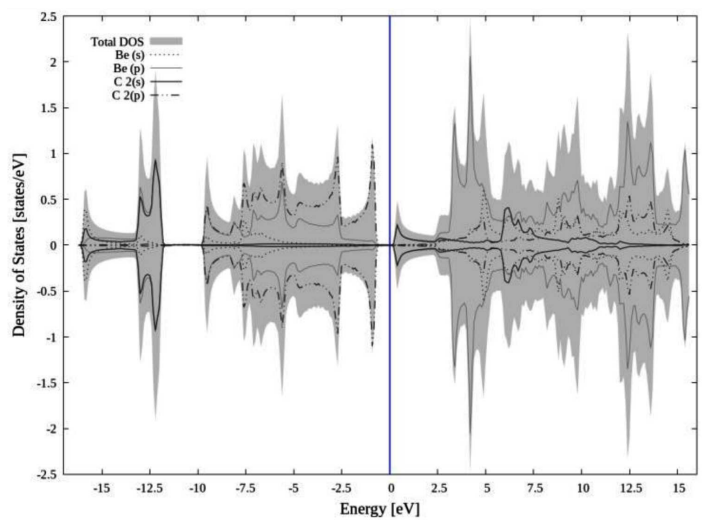

Fig. 3. Projected density of states of $\mathrm{CBe}_{2}$. The blue vertical line denotes the Fermi level.

As can be seen, there is a band gap of size $E_{g}=1.22 \mathrm{eV}$ visible both in the BAND and DOS plot which is of indirect type (between $\Gamma$ and $\mathrm{X}$ directions in the Brillouin zone). The presence of the band gap means that the material is semiconducting in nature. A shift in the Fermi level from the valence band $(\mathrm{VB})$ towards the conduction band (CB) typically indicates that the nature of this material is extrinsic-like, and that its nature is closer to an n-type semiconductor. This means that in comparison to the intrinsic state, there are more electrons in the conduction band than holes in VB (consequently, the electrons are majority charge carriers). In the next step, the projected density of states (PDOS) for the equilibrium lattice parameter was calculated and presented in Fig. 3.

The PDOS graph clearly shows the contribution of atomic shells shares into the total DOS (marked as the filled gray curve). It is clear that for the lowest lying states (near $-12.5 \mathrm{eV}$ ), the major contribution is coming from the carbon $(s)$ and beryllium $(p)$ shells. The major contribution in the -10 to $-2.5 \mathrm{eV}$ range comes from the $\mathrm{Be}(p)$ and $\mathrm{C}(p)$ shells, which suggests that they are important for the stabilization of $\mathrm{CBe}_{2}$ as the mixing in this range is strong. The highest occupied molecular orbital (HOMO) is almost purely contributed by the $\mathrm{C}(p)$ shell, yet there is a visible small contribution from the $\operatorname{Be}(p)$ states, also beneficial to bonding. The 


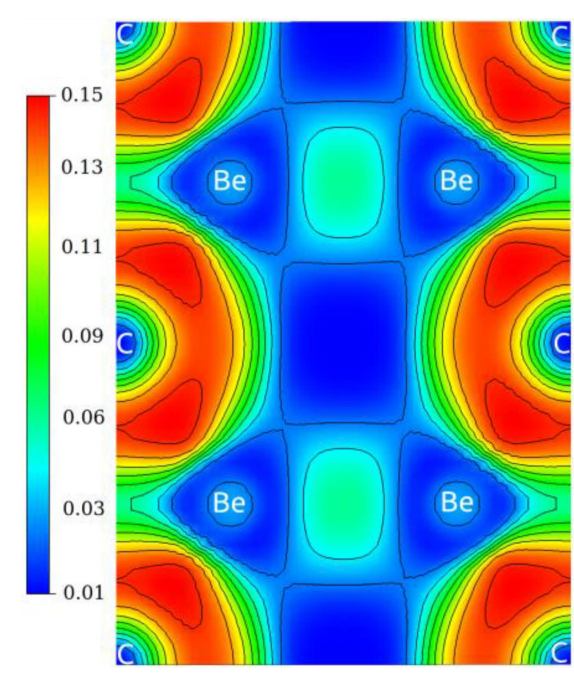

Fig. 4. Electron localization function for $(-110)$ plane of $\mathrm{CBe}_{2}$.

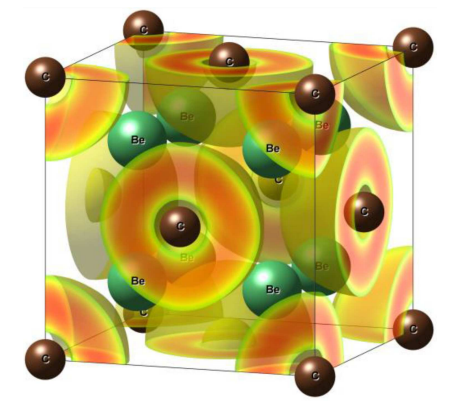

Fig. 5. Electron localization function of $\mathrm{CBe}_{2}$ conventional unit cell. The isovalue is set to $0.15 \mathrm{e} / \AA$.

lowest unoccupied molecular orbital (LUMO) consists mainly of evenly mixed $\mathrm{Be}(p)$ and $\mathrm{C}(s)$ while the remaining virtual orbitals are dominated by the $p$ shell coming from beryllium.

To determine the nature of the binding, the electron localization function (ELF) was calculated and presented in Fig. 4. For the preparation of this figure, a $(-110)$ cut plane was chosen, as this plane goes through the cross section of the largest possible number of atoms. Please note that only valence electrons are included in this plot. The analysis clearly shows that the electrons are mostly localized in the close vicinity of carbon ions with the maximal localization point located in between carbon and beryllium, which suggests an ionic type of bonding between $\mathrm{C}$ and Be. The blue regions in Fig. 4 localized near Be atoms also suggest that there is a significant charge transfer from Be ions. Additionally, there are visible localized electrons between the nearest Be (those aligned horizontally), making also a covalent bond. The charge between Be ions located above or below cannot be seen in the $(-100)$ plane, yet a similar analysis shows that this covalent character is also evident between each nearest Be ion. The overall cell ELF is presented in Fig. 5.
As can be seen in Fig. 5, the major ELF is in the close environment of carbon ions. At this surface, the isovalue level ELF between Be is not visible, which also confirms a weak covalent bonding between Be ions. To investigate it more precisely, the Bader charge analysis was carried out [16]. From this analysis, it is clearly seen that each Be is depleted of 1.97 electrons while each carbon gets nearly 1.94 electrons.

\section{Conclusions}

The structural and electronic properties of the $\mathrm{CBe}_{2}$ phase were calculated. The calculated bulk modulus of $180 \mathrm{GPa}$ means that the material is rather hard to compress. From the conducted electronic analysis, the semiconducting nature of the compound is revealed with the indirect band gap of $1.22 \mathrm{eV}$. The frontier molecular orbital, as shown in the PDOS figure, consists mainly of the C $2(p)$ shell which in connection with the Be $2(p)$ shell is mainly responsible for the stabilization due to strong mixing below the Fermi level. By utilizing the electron localization function, the nature of bonding was specified. The bonding between carbon and beryllium is of ionic nature, while the bonding between the nearest Be has a covalent character. The Bader charge analysis seems to confirm this observation, showing the additional charge accumulated at carbon ions while the charge is depleted of beryllium.

\section{Acknowledgments}

This research was partly supported by PL-Grid Infrastructure.

\section{References}

[1] P. Lebeau, Compt. rend. 121, 496 (1895).

[2] R.W.G. Wyckoff, Crystal Structures, 2nd ed., Interscience Publishers, New York 1963, p. 239.

[3] Y. Li, Y. Liao, Z. Chen, Angewandte 53, 7248 (2014).

[4] M. Naseri, J. Jalilian, F. Parandin, K. Salehi, Phys. Lett. A 382, 2144 (2018).

[5] Y. He, L. Jin, J. Zhang, B. Luo, K. Li, $\mathrm{W}$. Wu,J. Luo, Matter Radiat. Extremes 4, 045403 (2019).

[6] C. Hua-Liang, C. Xin-Lu, Z. Hong, Chin. Phys. B 29, 016601 (2020).

[7] B. Pullock, J. Phys. Chem. 63, 587 (1959).

[8] J. Coobs, W. Koshuba, J. Electrochem. Soc., 115 (1952).

[9] P. Tole, P. Fowler, Mol. Simul. 4, 331 (1990). 
[10] S. Reed, E. Funston, W. Bridges, Anal. Chim. Acta 10, 429 (1954).

[11] C. Bjorkas, N. Juslin, H. Timko, K. Voortler, K. Nordlund, K. Henriksson, P. Erhart, J. Phys.: Condens. Matter 21, 445002 (2009).

[12] M. Mehine, C. Bjorkas, K. Vorter, K. Nordlund, M.I. Airila, J. Nucl. Mater. 414, 1 (2011).

[13] Y. Ferro, A. Allouche, C. Linsmeier, J. App. Phys. 113, 213514 (2013).
[14] P. Giannozzi, O. Andreussi, T. Brumme et al., J. Phys.: Condens. Matter 29, 465901 (2017).

[15] K. Gruszka, K. Bednarska, Acta Phys. Pol. A 135, 107 (2019).

[16] W. Tang, E. Sanville, G. Henkelman, J. Phys.: Condens. Matter 21, 084204 (2009). 